\title{
Neural adaptive control for flocking of Agents with unknown nonlinear dynamics
}

\author{
QI Xiaowei ${ }^{*}$, Ren Guang \\ Dalian Maritime University, Liao-Ning China \\ *xiaowei0735@163.com, reng@dlmu.edu.cn
}

\begin{abstract}
Keywords: distributed control;neural networks; adaptive flocking ;unknown nonlinear dynamics; Abstract. This paper is concerned with the leader-follower flocking problem of networked nonholonomic multi-agent systems with non-identical unknown nonlinear dynamics. The leader motion to be synchronized is also non-linear and unknown. By employing the graph theory and a pinning control technique, a distributed neural adaptive control design is developed for the agents to achieve motion synchronization with the leader.The design is for a directed communication graph with a fixed topology. A collective potential function is used to maintain cohesion between the agents. On the basis of Lyapunov analysis, the developed neural flocking algorithm guarantees that all the agents' headings and speeds are synchronized with the leader and collisions between the agents can be avoided.An illustrative example is given to show the effectiveness of the proposed control strategy which may be applied to surveying in the civil engineering.
\end{abstract}

\section{Introduction}

Flocking is a coordinated motion of a large group of agents with local interactions. This issue has a historical background in physics, biology and computer science [1] and has attracted significant attention in the control and system community because of its broad applications in formation control of mobile robots, mobile sensor networks, cooperative coordination of unmanned aerial vehicles, cooperative search and rescue,coordinated exploration and exploitation. The key challenging problem of flocking control is how to design a distributed control law based on local interactions among agents so that collective behaviors can emerge [2]. The studies on collective behavior of multi-agent systems include consensus[3],synchronization and flocking[4], which may date back to1986, Reynolds giving the three rules to animate the flocking behavior as follows:separation,alignment and cohesion[5].

In recent years, many flocking algorithms have been proposed and stable flocking criteria have been obtained for second-order multi-agent systems, either under fixed topology,switching topology,or being considered with time delays. Olfati-saber provided a theoretical framework for the design and analysis of distributed flocking algorithm[6], which were on the basis of the assumption that all agents been informed by a virtual leader traveling at a constant velocity. Literature [7] studied the leaderless flocking problem of nonholonomic agents under undirected communication structure,proposing a synchronization strategy and avoiding collisions between the agents using local artificial potential fields. [8] studied the flocking and velocity alignment in a team of nonholonomic agents in two and three dimensions. A geodesic control law is developed that minimizes a misalignment potential and results in velocity alignment and flocking.In all of the aforementioned works, the dynamical model of the agents and possible uncertainties in their dynamics are not considered.

In this paper, a control design method is presented for robust adaptive flocking of nonholomonic multi-agent systems with an active leader.The agents have non-identical unknown nonlinear dynamics and are subject to disturbances from the environment.Moreover,the leader motion to be synchronized is also nonlinear and unknown. The control design is for directed communication structure with a fixed network topology. The developed flocking algorithm consists of a linear term, an augmented $\mathrm{NN}$ adaptive term and a gradient term of collective potential function, which can be implemented in a distributed manner. It is proved that with the developed neural flocking algorithm,the cooperative motion synchronization errors converge to a small neighborhood of origin 
and collisions between the agents can been avoided.An example is given to show the effectiveness of the proposed method.

\section{Problem formulation and preliminaries}

The flocking problem is stated as follows :given a team of $\mathrm{N}$ nonholonomic agents,design a control law $F_{i}$ and $\tau_{i}$ for the $i$ th agent such that all agents track the leader and the motion synchronization errors converge to a small neighborhood of origin. The local motion synchronization error is defined as

$$
\begin{aligned}
& e_{i \mathrm{v}}=\sum_{j \in \mathrm{N}_{i}} a_{i j}\left(v_{j}-v_{i}\right)+b_{i}\left(v_{0}-v_{i}\right) \\
& e_{i \psi}=\sum_{j \in \mathrm{N}_{i}} a_{i j}\left(\psi_{j}-\psi_{i}\right)+b_{i}\left(\psi_{0}-\psi_{i}\right) \\
& e_{i r}=\sum_{j \in \mathrm{N}_{i}} a_{i j}\left(r_{j}-r_{i}\right)+b_{i}\left(r_{0}-r_{i}\right)
\end{aligned}
$$

Neural flocking controller design. The flocking control problem confronted in this paper is to design a control strategy so that the local motion synchronization errors are bounded to a small neighborhood of origin and collisions between the agents can be avoided.Note that both the individual nonlinearities and disturbances are unknown.In addition ,the leader motion is also nonlinear and unknown. As such,the flocking controller must be robust to unknown individual dynamics and unknown leader dynamics. The control design follows three steps as velocity synchronization, angle synchronization, collision avoidance.

Step1:Velocity synchronization

The error vector of the $\mathrm{N}$ networked agents is given by

$e_{v}=-(L+B)\left(v-1 \otimes v_{0}\right)$

where $e_{v}=\left[e_{1 v}, \ldots, e_{N v}\right]^{T} \in R^{N}, v=\left[v_{1}, \ldots, v_{N}\right]^{T} \in R^{N}, B \in R^{N \times N}$ is the leader adjacency matrix.

Taking time derivative of $e_{v}$ gives

$$
\Leftrightarrow=-(L+B)\left[f_{v}(\bullet)+F+\omega_{v}(t)-1 \otimes f_{0 v}\left(v_{0}, t\right)\right]
$$

where $f_{v}(\bullet)=\left[f_{\mathrm{vv}}\left(v_{1}\right), \ldots, f_{N v}\left(v_{N}\right)\right]^{T} \in R^{N}, F=\left[F_{1}, \ldots, F_{N}\right]^{T} \in R^{N}, \omega_{v}(t)=\left[\omega_{\mathrm{vv}}(t), \ldots, \omega_{N v}(t)\right]^{T} \in R^{N}$.

Define the control input $F_{i}$ for the $i$ th agent as

$F_{i}=k_{i v} e_{i v}-f_{i v}\left(v_{i}\right)+\mu_{i}^{\prime}$

By some calculation, the following equation was finally got

$e_{v}=-(L+B)\left[\tilde{W}_{v} \varphi(v)+\varepsilon_{v}-1 \otimes f_{0 v}+k_{v} e_{v}+\mu^{\prime}(t)+\omega_{v}\right]$

where $f_{i v}\left(v_{i}\right)=W_{i v}^{T} \varphi\left(v_{i}\right)+\varepsilon_{i v}$ is an unknown function being approximated by an NN.The input control is with $\mu_{i}^{\prime}$ being an auxiliary input to be designed for collision avoidance and cohesion in the group. $\hat{f}_{i v}\left(v_{i}\right)$ is an estimate of $f_{i v}\left(v_{i}\right)$ which takes the NN weights vector $\hat{W}_{i v}^{T} \varphi_{i}\left(v_{i}\right) \in R^{n}$ and the adaptive law for $\hat{W}_{i v}^{T} \varphi_{i}\left(v_{i}\right)$ is $\underset{W_{i v}}{\&}=-\Gamma_{i F}\left[\varphi_{i} e_{i v}^{T} p_{i}\left(d_{i}+b_{i}\right)+k_{F} \hat{W}_{i v}\right]$.It is noticed that the variables are matrix form. $\mu^{\prime}=\left[\mu_{1}^{\prime}, \ldots, \mu_{N}^{\prime}\right]^{T} \in R^{N}, k v=\operatorname{diag}\left\{k_{i v}, \ldots, k_{N_{v}}\right\} \in R^{N \times N}, \varepsilon_{v}=\left[\varepsilon_{1 v}, \ldots, \varepsilon_{N v}\right]^{T} \in R^{N}, \varphi(v)=\left[\varphi_{1}^{T}\left(v_{1}\right), \ldots, \varphi_{N}^{T}\left(v_{N}\right)\right]^{T}$, $\hat{W}_{v}^{T}=\operatorname{diag}\left\{\hat{W}_{\mathrm{lv}}^{T}, \ldots, \hat{W}_{N v}^{T}\right\}, W_{v}^{T}=\operatorname{diag}\left\{W_{10}^{T}, \ldots, W_{N v}^{T}\right\}$, and $\tilde{W}_{v}=W_{v}-\hat{W}_{v}$.

Step2:Angle synchronization

The design of goal is to achieve the angle synchronization of the $\mathrm{N}$ agents with the leader.

Similar to the step 1,the equation of error vector of the $\mathrm{N}$ networked agents can be expressed by

$e_{\psi}=-(L+B)\left(\psi-\mathbf{1} \otimes \psi_{0}\right)$

$e_{r}=-(L+B)\left(r-\mathbf{1} \otimes r_{0}\right)$

The error dynamics can be obtained as

$\&_{s}=-(L+B)\left[\left(W_{r}-\hat{W}_{r}\right) \varphi(v)+\varepsilon_{r}-1 \otimes f_{0 r}+k_{s} e_{s}+\mu^{\prime \prime}+\lambda\left(r-1 \otimes r_{0}\right)\right]$

Step 3:Collision avoidance 
The collision avoidance among the agents is achieved by a bounded artificial potential function as follows

$$
\begin{aligned}
& V_{i j}\left(\left\|p_{i j}\right\|_{\sigma}\right)=\int_{d}^{p_{i j} \|_{\sigma}} \theta(s) d(s) \\
& \theta(z)=\omega_{h}(z / d)\left[z(z-d) / \sqrt{1+Z^{2}}-1\right]
\end{aligned}
$$

By rearranging and substitution, the error dynamics equation $\mathrm{s}$ are finally obtained as

\& $=-(L+B)\left[\tilde{W}_{v} \varphi(v)+\varepsilon_{v}-1 \otimes f_{0 v}+k_{v} e_{v}+(\nabla V)_{\|}\left|e_{v}\right|+\omega_{v}\right]$

$$
\oint_{s}=-(L+B)\left[\left(W_{r}-\hat{W}_{r}\right) \varphi(v)+\varepsilon_{r}-\mathbf{1} \otimes f_{0 r}+k_{s} e_{s}+(\nabla V)_{\perp}\left|e_{s}\right|+\omega_{r}+\lambda\left(r-\mathbf{1} \otimes r_{0}\right)\right]
$$

\section{Neural flocking controller analysis.}

Assumption1:(1)The unknown disturbances $\omega_{i r}$ and $\omega_{i v}$ of the $i$ th agent are bounded.The overall disturbance vectors $\omega_{v}$ and $\omega_{r}$ are bounded by $\left\|\omega_{v}\right\| \leq \omega_{v M},\left\|\omega_{r}\right\| \leq \omega_{r M}$, where the $\omega_{v M}, \omega_{r M}$ are positive constants.

Proof:consider the following Lyapunov function candidate

$$
H=\frac{1}{2} e_{v}^{T} P e_{v}+\frac{1}{2} e_{s}^{T} P e_{s}+\frac{1}{2} \operatorname{tr}\left\{\tilde{W}_{v}^{T} \Gamma_{F}^{-1} \tilde{W}_{v}\right\}+\frac{1}{2} \operatorname{tr}\left\{\tilde{W}_{r}^{T} \Gamma_{\tau}^{-1} \tilde{W}_{r}\right\}+\frac{1}{2} e_{\psi}^{T} e_{\psi}
$$

Taking the time derivative of $H$ and taking the error dynamics (11)(12)and the adaptive laws $\underset{W_{i r}}{\&}, W_{i v}^{\&}, \mu_{i}{ }^{\prime}, \mu_{i}{ }^{\prime \prime}$ into (13), the equation (14) can be finally got by using Young's inequality.

$$
\begin{aligned}
& H(t) \leq H(0) e^{-\alpha t}+\frac{\beta}{\alpha}\left(1-e^{-\alpha t}\right) \\
& \alpha:=2 \min \left\{\frac{\frac{k_{F}}{2}-\varphi_{M} \bar{\rho}(P) \bar{\rho}(A)}{\bar{\rho}\left(\Gamma_{F}^{-1}\right)}, \frac{\frac{k_{\tau}}{2}-\varphi_{M} \bar{\rho}(P) \bar{\rho}(A)}{\bar{\rho}\left(\Gamma_{\tau}^{-1}\right)}, \frac{\frac{1}{2} \lambda_{\min }\left(k_{v}\right) \underline{\rho}(Q)-\left(\vartheta_{M}+B_{1 M}\right) \bar{\rho}(P) \bar{\rho}(L+B)-\frac{1}{4} \varphi_{M} \bar{\rho}(P) \bar{\rho}(A)}{\bar{\rho}(P)},\right. \\
& \left.\frac{\frac{1}{2} \lambda_{\min }\left(k_{s}\right) \underline{\rho}(Q)-\left(\vartheta_{M}+B_{2 M}\right) \bar{\rho}(P) \bar{\rho}(L+B)-\frac{1}{4} \varphi_{M} \bar{\rho}(P) \bar{\rho}(A)-\frac{1}{4}-\bar{\rho}(P) \bar{\rho}(\lambda)-\frac{1}{4} \bar{\rho}(P) \bar{\rho}^{2}(\lambda)}{\bar{\rho}(P)}, \underline{\rho}(\lambda)-1-\bar{\rho}(P) \bar{\rho}^{2}(\lambda)\right\}
\end{aligned}
$$

and

$$
\beta:=\frac{1}{4} \bar{\rho}(P) \bar{\rho}(L+B) B_{2 M}+\frac{1}{4} \bar{\rho}(P) \bar{\rho}(L+B) B_{1 M}+\frac{k_{F}}{2}\left\|W_{F}\right\|_{F}^{2}+\frac{k_{\tau}}{2}\left\|W_{\tau}\right\|_{F}^{2}
$$

Thus, the cooperative tracking errors $\left\|e_{r}\right\|,\left\|e_{v}\right\|,\left\|e_{\psi}\right\|$ converge to a compact set $\Omega_{\psi}=\left\{\left\|e_{\psi}\right\| \leq \sqrt{\frac{2 \beta}{\alpha}}\right\}, \Omega_{v}=\left\{\left\|e_{v}\right\| \leq \sqrt{\frac{2 \beta}{\underline{\rho}(P) \alpha}}\right\}, \Omega_{r}=\left\{\left\|e_{r}\right\| \leq \sqrt{\frac{2 \beta}{\alpha}}\right\}$ respectively .It can be made arbitrarily small by appropriately choosing the gains $\Gamma_{i F}, \Gamma_{i \tau}, \lambda_{i}$. That is the proof.

Example. Consider a five node graph with a leader connected to agents1and 4. The Laplacian matrix $L$ is given by $[2,-1,0,0,-1 ; 0,2,0,-1,-1 ; 0,0,1,0,-1 ; 0,-1,0,1,0 ;-1,0,-1,0,2]$ and the leader adjacency matrix $B$ is $\operatorname{diag}\{1,0,0,10\}$. Let the state vector for the $i$ th agent be $\left[x_{i}, y_{i}, \psi_{i}, v_{i}, r_{i}\right]^{T}$. The initial states for the five agents are given by $A_{1}=[-2,0,0,0,0]^{T}, A_{2}=[2,0, \pi / 6,4,1]^{T}$, $A_{3}=[0,-2,-\pi / 3,-2,0]^{T}, A_{4}=[0,1, \pi / 3,-2,-2]^{T}, A_{5}=[-1,-3,-\pi / 4,-2,2]^{T}$, the leader initial state is $[0,0, \pi / 4,2,0]$.

In the simulation ,the parameters are chosen as $k_{i v}=k_{i s}=32, \Gamma_{i F}=\Gamma_{i \tau}=250, k_{F}=k_{\tau}=0.1, \lambda_{i}=2$. The centers of Gaussian functions are chosen as $\{-5,0,5\} \times\{-2,0,2\}$ and the initial weights are 0 .We consider the case where the leader travels at a constant velocity,then at a varying velocity. In both cases, we will show that flocking behavior can be observed although disturbed by the unknown individual dynamics and unknown leader dynamics.Figure1 shows the entire flocking phenomenon of five nonholonomic agents with a leader in $v_{0}=2+0.9 \sin (2.3 t), \psi_{0}=\pi / 4+0.5 \sin (2 t)$ and information exchange topology matrix $L$.We can observe that the formation is well-established and no collision between agents occurred despite the existence of unknown individual dynamics.Figure 2 demonstrates the states of the five agents, and they are synchronized with the leader after 4 seconds. 


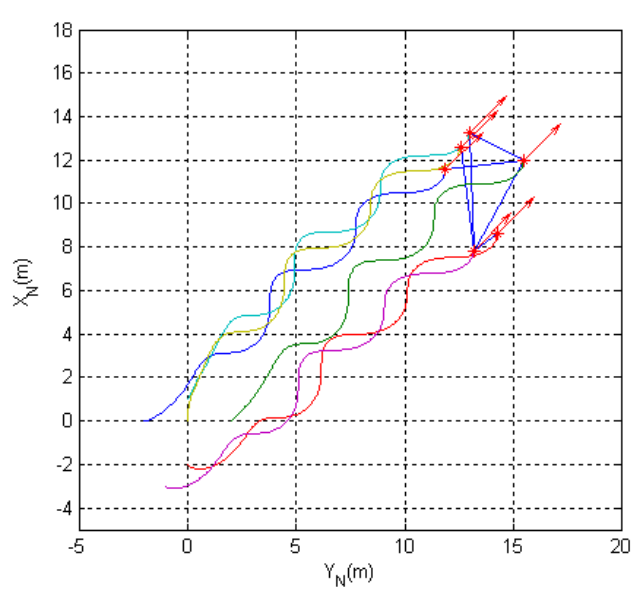

Fig. 1 Flocking path of the agents
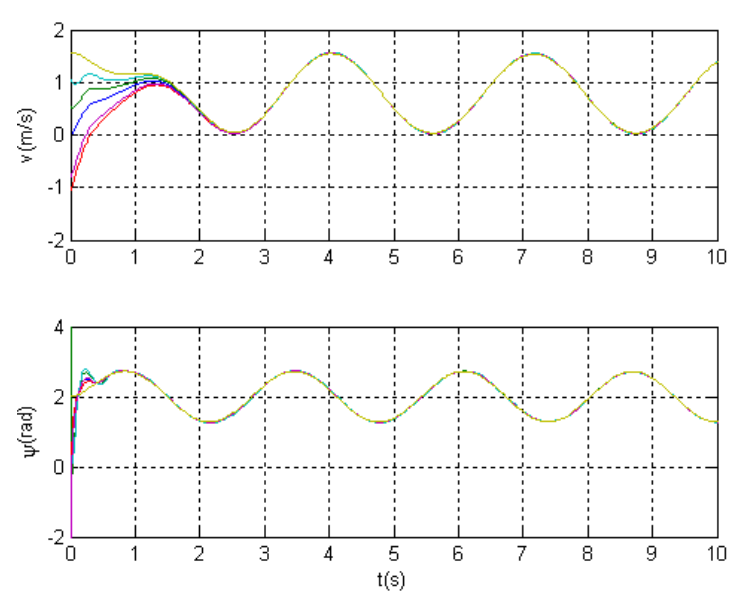

Fig. 2 State synchronization of the agents

\section{Summary}

This paper presented a neural adaptive control design for the leader-follower flocking of nonholonomic multi-agent systems with unknown individual dynamics and unknown leader dynamics. The development is for the general directed communication structure with a fixed topology.On the basis of Lypunov theory, the developed neural flocking algorithm gurantees that the local synchronization errors converge to a small neighborhood of origin.Collisions between the agents can be avoided using artificial potential function. Simulation results demonstrated the effectiveness of the neural flocking controller and the leaning ability of NNs.

\section{References}

[1] H. Levine, W.J. Rappel, Self organization in systems of self-propelled particles,J, Physical Review E 63 (2001) 208-21

[2] C.W.Reynolds,Flocks, herds, and schools:a distributed behavioral model,J.Computer Graphics. 21 (1987) 25-34

[3] S.Housheng, Z.Ningzi and Z.Michael Adaptive flocking with a virtual leader of multiple agents governed by locally Lipschitz nonlinearity,J..Nonlinear analysis.14(2013) 798-806.

[4] B.Michael,W.Lennart Pyritz, B Margarete.Spontaneous flocking in human groups,J. Behavioral Processes 92(2013) 6-14

[5] J. van der Geer, J.A.J. Hanraads, .A. Lupton, The art of writing a scientific article, J. Sci. Commun. 163 (2000) 51-59.

[6] R Olfati-saber,RM Murray,Consensus problems in networks of agents with switching topology and time-delays,J.IEEE Transactions on Automatic Control 49(2007) 1522-1533

[7] HG Tanner, A Jadbabaie ,GJ.Pappas,Flocking in teams of nonholonomic agents,J. Lecture Notes in Control and Information Sciences 309(2005) 458-460

[8] W. Ni, D. Cheng, Leader-following consensus of multi-agent systems under fixed and switching topologies, Systems \& Control Letters 59 (3-4) (2010) 209-217 